\title{
FUZZY IMPLICATIVE IDEALS IN BCK-ALGEBRAS
}

\author{
Y. B. JUN, S. M. HONG AND E. H. ROH
}

Abstract. An implicative ideal of a BCK-algebra is defined by Iséki [1]. In 1991, $\mathrm{Xi}$ [7] defined a fuzzy implicative ideal of a BCK-algebra. In this paper, we investigate the properties of fuzzy implicative ideals in BCK-algebras.

The concept of fuzzy sets was introduced by Zadeh in [8]. Rosenfeld [6] applied it to the fundamental theory of groups. In [7], Xi applied the concept of fuzzy sets to BCKalgebras, and he got some interesting results. The aim of this paper is to investigate the properties of fuzzy implicative ideals in BCK-algebras.

Let us recall some definitions and results, which are necessary for development of the paper.

An algebra $(X ; *, 0)$ of type $(2,0)$ is called a BCK-algebra if it satisfies the following conditions:

$\mathrm{BCK}-1(x * y) *(x * z) \leq z * y$,

$\mathrm{BCK}-2 x *(x * y) \leq y$,

BCK $-3 x \leq x$,

BCK-4. $0 \leq x$,

$\mathrm{BCK}-5 x \leq y$ and $y \leq x$ imply $x=y$,

$\mathrm{BCK}-6 x \leq y$ if and only if $x * y=0$,

for all $x, y, z \in X$.

In any $\mathrm{BCK}$-algebra $X$, the following properties hold:

(1) $(x * y) * z=(x * z) * y$,

(2) $x * 0=x$,

(3) $x * y \leq x$,

(4) $(x * z) *(y * z) \leq x * y$,

(5) $x \leq y$ implies $x * z \leq y * z$ and $z * y \leq z * x$.

Received September 23, 1993; revised January 6, 1994.

1991 Mathematics Subject Classification. 03G25, 06F35, 94D05.

Key words and phrases. Implicative ideal, fuzzy implicative ideal, level implicative ideal. 
A fuzzy set $\mu$ in a set $X$ is a function from $X$ into $[0,1]$. For a fuzzy set $\mu$ in $X$ and $t \in[0,1]$, the set

$$
\mu_{t}=\{x \in X: \mu(x) \geq t\}
$$

is called a level subset of $\mu$.

In what follows, $X$ would mean a BCK-algebra unless otherwise specified.

Definition 1 ([1]). A nonempty subset $I$ of $X$ is said to be implicative if it satisfies

(P1) $0 \in I$,

(P2) $(x * y) * z \in I$ and $y * z \in I$ imply $x * z \in I$ for all $x, y, z \in X$.

Definition 2 ([7]). A fuzzy set $\mu$ in $X$ is called a fuzzy implicative ideal (briefly, a f.i. ideal) of $X$ if

(F1) $\mu(0) \geq \mu(x)$ for all $x \in X$,

(F2) $\mu(x * z) \geq \min \{\mu((x * y) * z), \mu(y * z)\}$ for all $x, y, z \in X$.

Example 1. Let $I$ be an implicative ideal of $X$ and let $\mu$ be a fuzzy set in $X$ defined by

$$
\mu(x)= \begin{cases}0 & \text { if } x \notin I, \\ t & \text { if } x \in I,\end{cases}
$$

where $t$ is a fixed number in $(0,1)$. Then $\mu$ is a $f . i$. ideal of $X$. In fact, it is clear that $\mu(0) \geq \mu(x)$ for all $x \in X$. In order to prove that $\mu$ satisfies (F2), we will divide into the following cases:

If $(x * y) * z \in I$ and $y * z \in I$, then $x * z \in I$. Thus $\mu(x * z)=\mu((x * y) * z)=\mu(y * z)=t$, and so

$$
\mu(x * z) \geq \min \{\mu((x * y) * z), \mu(y * z)\} .
$$

If $(x * y) * z \notin I$ and $y * z \notin I$, then $\mu((x * y) * z)=\mu(y * z)=0$. Hence

$$
\mu(x * z) \geq \min \{\mu((x * y) * z), \mu(y * z)\}
$$

If exactly one of $(x * y) * z$ and $y * z$ belongs to $I$, then exactly one of $\mu((x * y) * z)$ and $\mu(y * z)$ is equal to 0 . Thus

$$
\mu(x * z) \geq \min \{\mu((x * y) * z), \mu(y * z)\} .
$$

Consequently $\mu$ is a $f . i$. ideal of $X$.

Theorem 1 ([7]). Let $\mu$ be a fuzzy set in $X$. Then $\mu$ is a f.i. ideal of $X$ if and only if $\mu_{t}$ is an implicative ideal of $X$ for all $t \in[0.1]$, when $\mu_{t} \neq \varnothing$.

Definition 3. Let $\mu$ be a $f . i$. ideal of $X$. The implicative ideals $\mu_{t}, t \in[0,1]$, are called level implicative ideals of $X$. 
Note that if $X$ is a finite BCK-algebra, then the number of implicative ideals of $X$ is finite whereas the number of level implicative ideals of a $f . i$. ideal $\mu$ appears to be infinite. But, since every level implicative ideal is indeed an implicative ideal of $X$, not all these level implicative ideals are distinct. The next theorem characterizes this aspect.

Theorem 2. If $\mu$ is a f.i. ideal of $X$, then two level implicative ideals $\mu_{t_{1}}$ and $\mu_{t_{2}}\left(\right.$ with $\left.t_{1}<t_{2}\right)$ of $\mu$ are equal if and only if there is no $x \in X$ such that $t_{1} \leq \mu(x)<t_{2}$.

Proof. Assume that $\mu_{t_{1}}=\mu_{t_{2}}$ for $t_{1}<t_{2}$ and that there exists $x \in X$ such that $t_{1} \leq \mu(x)<t_{2}$. Then $\mu_{t_{2}}$ is a proper subset of $\mu_{t_{1}}$, which is impossible.

Conversely suppose that there is no $x \in X$ such that $t_{1} \leq \mu(x)<t_{2}$. Note that $t_{1}<t_{2}$ implies $\mu_{t_{2}} \subseteq \mu_{t_{1}}$. If $x \in \mu_{t_{1}}$, then $\mu(x) \geq t_{1}$. Since $\mu(x) \nless t_{2}$, it follows that $\mu(x) \geq t_{2}$, so that $x \in \mu_{t_{2}}$. This shows that $\mu_{t_{1}}=\mu_{t_{2}}$. This completes the proof.

Remark 1. As a consequence of Theorem 2, the level implicative-ideals of a $f . i$. ideal $\mu$ of a finite $\mathrm{BCK}$-algebra $X$ form a chain. But $\mu(0) \geq \mu(x)$ for all $x \in X$. Therefore $\mu_{t_{0}}$, where $t_{0}=\mu(0)$, is the smallest level implicative ideal but not always $\mu_{t_{0}}=\{0\}$ as shown in the following example, and hence we have the chain:

$$
\mu_{t_{0}} \subset \mu_{t_{1}} \subset \cdots \subset \mu_{t_{r}}=X
$$

where $t_{0}>t_{1}>\ldots>t_{r}$.

Notation. $\operatorname{Im}(\mu)$ denotes the image set of $\mu$.

Example 2. Let $I$ be a nonzero implicative ideal of $X$ and let $\mu$ be the $f . i$. ideal of $X$ as in Example 1. Then $\operatorname{Im}(\mu)=\{0, t\}$. Further, the two level implicative ideals of $\mu$ are $\mu_{0}=X$ and $\mu_{t}=I$. Thus we have that $\mu(0)=t$ but $\mu_{t}=I \neq\{0\}$.

Theorem 3. Let $\mu$ be a fi. ideal of $X$. If $\operatorname{Im}(\mu)=\left\{t_{1}, t_{2}, \ldots, t_{n}\right\}$, where $t_{1}<$ $t_{2}<\ldots<t_{n}$, then the family of implicative ideals $\mu_{i_{i}}(i=1,2, \cdots, n)$ constitutes all the level implicative ideals of $\mu$.

Proof. Let $t \in[0,1]$ and $t \notin \operatorname{Im}(\mu)$. If $t<t_{1}$, then $\mu_{t_{1}} \subseteq \mu_{t}$. Since $\mu_{t_{1}}=X$, therefore $\mu_{t}=X$ and $\mu_{t}=\mu_{t_{1}}$. If $t_{i}<t<t_{i+1}(1 \leq i \leq n-1)$, then there is no $x \in X$ such that $t \leq \mu(x)<t_{i+1}$. It follows from Theorem 2 that $\mu_{t}=\mu_{t_{i+1}}$. This shows that for any $t \in[0,1]$, the level implicative ideal $\mu_{t}$ is in $\left\{\mu_{t_{i}}: i=1,2, \ldots, n\right\}$.

Lemma. 1. Let $\mu$ be a f.i. ideal of a finite BCK-algebra $X$. If s and $t$ belong to $\operatorname{Im}(\mu)$ such that $\mu_{s}=\mu_{t}$, then $s=t$.

Proof. Assume that $s \neq t$, say $s<t$. Then there is $x \in X$ such that $\mu(x)=s<t$, and so $x \in \mu_{s}$ and $x \notin \mu_{t}$. Thus $\mu_{s} \neq \mu_{t}$, a contradiction. The proof is complete. 
Theorem 4. Let $\mu$ and $\nu$ be two f.i. ideals of a finite BCK-algebra $X$ with identical family of level implicative ideals. If $\operatorname{Im}(\mu)=\left\{t_{1}, t_{2}, \cdots, t_{m}\right\}$ and $\operatorname{Im}(\nu)=\left\{s_{1}, s_{2}, \ldots, s_{n}\right\}$, where $t_{1}>t_{2}>\ldots>t_{m}$ and $s_{1}>s_{2}>\cdots>s_{n}$, then

(a) $m=n$;

(b) $\mu_{i_{i}}=\nu_{s_{i}}, i=1, \ldots, m$;

(c) if $x \in X$ such that $\mu(x)=t_{i}$ then $\nu(x)=s_{i}, i=1, \ldots, m$.

Proof. By means of Theorem 3, we know that the only level implicative ideals of $\mu$ and $\nu$ are $\mu_{t_{i}}$ and $\nu_{s_{i}}$, respectively. Since $\mu$ and $\nu$ have the identical family of level implicative ideals, it follows that $m=n$. Thus (a) holds. Using Theorem 3 again, we get that $\left\{\mu_{t_{1}}, \ldots, \mu_{t_{m}}\right\}=\left\{\nu_{s_{1}}, \ldots, \nu_{s_{n}}\right\}$, and by Theorem 2 we have

$$
\mu_{t_{1}} \subset \mu_{t_{2}} \subset \cdots \subset \mu_{t_{m}}=X \text { and } \quad \nu_{s_{1}} \subset \nu_{s_{2}} \subset \cdots \subset \nu_{s_{n}}=X
$$

Hence $\mu_{t_{i}}=\nu_{s_{i}}, i=1, \cdots, m$ and (b) holds.

Let $x \in X$ be such that $\mu(x)=t_{i}$ and let $\nu(x)=s_{j}$. Noticing that $x \in \nu_{s_{i}}$, that is, $\nu(x) \geq s_{i}$, we obtain $s_{j} \geq s_{i}$. Thus $\nu_{s_{j}} \subseteq \nu_{s_{i}}$. Since $x \in \nu_{s_{j}}$ and $\nu_{s_{j}}=\mu_{i_{j}}$, therefore $x \in \mu_{t_{j}}$ and so $t_{i}=\mu(x) \geq t_{j}$. It follows that $\mu_{t_{i}} \subseteq \mu_{t_{j}}$. By (b), $\nu_{s_{i}}=\mu_{t_{i}} \subseteq \mu_{t_{j}}=\nu_{s_{j}}$. Consequently $\nu_{s_{i}}=\nu_{s_{j}}$, and by Lemma 1 we conclude that $s_{i}=s_{j}$. Thus $\nu(x)=s_{i}$. The proof is complete.

Theorem 5. Let $\mu$ and $\nu$ be two f.i. ideals of a finite BCK-algebra $X$ such that the families of level implicative ideals of $\mu$ and $\nu$ are identical. Then $\mu=\nu$ if and only if $\operatorname{Im}(\mu)=\operatorname{Im}(\nu)$.

Proof. $(\Rightarrow)$ It is clear.

$(\Leftarrow)$ Assume that $\operatorname{Im}(\mu)=\operatorname{Im}(\nu)=\left\{t_{1}, \ldots, t_{n}\right\}$ where $t_{1}>\cdots>t_{n}$. Let $x_{1}, \ldots, x_{n}$ be distinct elements of $X$ such that $\mu\left(x_{i}\right)=t_{i}(1 \leq i \leq n)$. By Theorem $4(\mathrm{c}), \nu\left(x_{i}\right)=$ $t_{i}(1 \leq i \leq n)$. Since for any $x \in X$ there exists some $t_{i}$ such that $\mu(x)=t_{i}$, therefore $x \in \mu_{t_{i}}$. Hence $\nu(x) \geq t_{i}=\mu(x)$. By the same argument, we obtain $\mu(x) \geq \nu(x)$. Consequently $\mu(x)=\nu(x)$ for all $x \in X$. This completes the proof.

Theorem 6. Let $X$ be a finite BCK-algebra and let $\mu$ be a fuzzy set in $X$ with $\operatorname{Im}(\mu)=\left\{t_{o}, t_{1}, \cdots, t_{k}\right\}$ where $t_{o}>t_{1}>\ldots>t_{k}$. If there exists a chain of implicative ideals of $X$ :

$$
I_{0} \subset I_{1} \subset \cdots \subset I_{k}=X
$$

such that $\mu\left(\bar{I}_{n}\right)=t_{n}$, where $\bar{I}_{n}=I_{n}-I_{n-1}, I_{-1}=\varnothing, n=0,1, \ldots, k$, then $\mu$ is a f.i. ideal of $X$.

Proof. Since $0 \in I_{0}$, we have $\mu(0)=t_{0} \geq \mu(x)$ for all $x \in X$. We divide into the following cases to prove that $\mu$ satisfies (F2): If $(x * y) * z \in \bar{I}_{n}$ and $y * z \in \bar{I}_{n}$, then $x * z \in I_{n}$ because $I_{n}$ is an implicative ideal of $X$. Thus 


$$
\mu(x * z) \geq t_{n}=\min \{\mu((x * y) * z), \mu(y * z)\}
$$

If $(x * y) * z \notin \bar{I}_{n}$ and $y * z \notin \bar{I}_{n}$, then the following four cases arise:

1. $(x * y) * z \in X-I_{n}$ and $y * z \in X-I_{n}$,

2. $(x * y) * z \in I_{n-1}$ and $y * z \in I_{n-1}$,

3. $(x * y) * z \in X-I_{n}$ and $y * z \in I_{n-1}$,

4. $(x * y) * z \in I_{n-1}$ and $y * z \in X-I_{n}$.

But, in either case, we know that

$$
\mu(x * z) \geq \min \{\mu((x * y) * z), \mu(y * z)\}
$$

If $(x * y) * z \in \bar{I}_{n}$ and $y * z \notin \bar{I}_{n}$, then either $y * z \in I_{n-1}$ or $y * z \in X-I_{n}$. It follows that either $x * z \in I_{n}$ or $x * z \in X-I_{n}$. Thus

$$
\mu(x * z) \geq t_{n}=\min \{\mu((x * y) * z), \mu(y * z)\}
$$

If $(x * y) * z \notin \bar{I}_{n}$ and $y * z \in \bar{I}_{n}$, then by similar process we have

$$
\mu(x * z) \geq \min \{\mu((x * y) * z), \mu(y * z)\}
$$

Summarizing the above results, we obtain

$$
\mu(x * z) \geq \min \{\mu((x * y) * z), \mu(y * z)\}
$$

for all $x, y, z \in X$. Consequently $\mu$ satisfies the condition (F2). This complietes the proof.

Theorem 7. Let $\mu$ be a f.i. ideal of a finite $B C K$-algegra $X$. If $\operatorname{Im}(\mu)=$ $\left\{t_{0}, t_{1}, \ldots, t_{k}\right\}$ where $t_{o}>t_{1}>\cdots>t_{k}$, then

(a) $I_{n}=\mu_{t_{n}}(n=0,1, \ldots, k)$ is an implicative ideal of $X$,

(b) $\mu\left(\bar{I}_{n}\right)=t_{n}(n=0,1, \ldots, k)$ where $\bar{I}_{n}=I_{n}-I_{n-1}$ and $I_{-1}=\varnothing$,

Proof. (a) is by Theorem 3.

(b) Obviously $\mu\left(I_{0}\right)=t_{0}$. Since $\mu\left(I_{1}\right)=\left\{t_{0}, t_{1}\right\}$, for $x \in \bar{I}_{1}$ we have $\mu(x)=t_{1}$. Hence $\mu\left(\bar{I}_{1}\right)=t_{1}$. Repeating the above argument, we have $\mu\left(\bar{I}_{n}\right)=t_{n}(n=0,1, \ldots, k)$. This completes the proof.

\section{Acknowledgement}

We are grateful to the referee for valuable suggestions and comments. 


\section{References}

[1] K. Iséki, "On ideals in BCK-algebras," Math. Seminar Notes (Presently, Kobe J. Math), 3 (1975), 1-12.

[2] K. Iséki and S. Tanaka, "Ideal theory of BCK-algebras," Math. Japon., 21 (1976), 351-366.

[3] Y. B. Jun, "Characterizations of fuzzy ideals by their level ideals in BCK(BCI)-algebras," Math. Japon., 38 (1993), 67-71.

[4] Y. B. Jun, S. M. Hong, J. Meng and X. L. Xin, "Characterizations of fuzzy positive implicative ideals in BCK-algebras," Math. Japon., 40 (1994), 503-507.

[5] J. Meng, "Ideals in BCK-algebras (Chinese)," Pure and Appl. Math., 2 (1986), 68-76.

[6] A. Rosenfeld, "Fuzzy groups," J. Math. Anal. Appl., 35 (1971), 512-517.

[7] O. G. Xi, "Fuzzy BCK-algebras," Math. Japon., 36 (1991), 935-942.

[8] L. A. Zadeh, "Fuzzy sets," Inform. Control, 8 (1965), 338-353.

Department of Mathematics, Gyeongsang National University, Chinju 660-701, Korea. 